\title{
LA COMPETENCIA DE TRABAJO EN EQUIPO: MÁS ALLÁ DEL CORTA Y PEGA
}

\section{COMPETITION TEAM WORK: BEYOND THE CUT AND \\ PASTE}

\begin{abstract}
AUTOR/ES
Javier Barraycoa Martínez: Co-director del Observatorio Laboral de la Universitat Abat Oliba CEU.

Catalunya (España).jbarrayc@uao.es

Olga Lasaga Millet: Co-directora del Observatorio Laboral de la Universitat Abat Oliba CEU. Catalunya (España). olasaga@uao.es
\end{abstract}

\section{RESUMEN}

La formación en muchas de las competencias profesionales empieza en la escuela. Ello hace indispensable consensuar su definición y contenido a fin de evitar disonancias entre el entorno educativo y el mercado de trabajo. En el presente estudio se aborda el análisis y redimensión de una de las competencias más valoradas por las empresas: el trabajo en equipo.

\section{PALABRAS CLAVE}

Competencias - Trabajo en equipo - Mercado laboral

\section{ABSTRACT}

Training in many of the skills begins at school. This makes it essential to agree the definition and content to avoid dissonance between the educational environment and the labour market. This study presents the analysis and a resizing of skills most valued by business: teamwork.

\section{KEY WORDS}

Skills - Teamwork - Labour market

ÍNDICE

\section{INTRODUCCIÓN}

2. PARADOJAS

3. REDEFINICIÓN DE LA COMPETENCIA TRABAJO EN EQUIPO

$\sim$ 4. DEL TRABAJO EN GRUPO AL TRABAJO EN EQUIPO

^. BIBLIOGRAFÍA 


\section{INTRODUCCIÓN}

En los últimos tiempos se ha ido popularizando toda una terminología educativa en relación a las competencias. Escuelas y universidades centran parte de sus recursos pedagógicos en preparar a sus alumnos en las habilidades y aptitudes que en un futuro les reclamará el mercado laboral. Sin embargo, los estudios sobre inserción laboral revelan que con frecuencia aparecen disonancias entre el significado que atribuyen los empleadores a algunas competencias respecto del sentido otorgado a las mismas en el ámbito académico. En este artículo se aborda la problemática conceptual de una competencia especialmente valorada por los empleadores: el trabajo en equipo. Se trata de una competencia transversal (aplicable a todo tipo de titulaciones) que se caracteriza por su dimensión relacional.

\section{PARADOJAS}

En principio, entre la Universidad y la Empresa, parece haber un acuerdo sobre la importancia y alcance de esta competencia. El trabajo en equipo es la competencia transversal más solicitada por los empleadores y, además, los licenciados reconocen que es la más utilizada en el desempeño diario del puesto de trabajo, según confirman numerosas investigaciones cuantitativas de carácter general (Gómez Gras et al., 2003; Pastor et al., 2005; Fundación Universidad Carlos III, 2005, Informe Reflex, 2007). Sin embargo al profundizar en su análisis se descubren algunas paradojas.

En primer lugar, en los mencionados estudios se desprende que es la competencia en la que los recién licenciados afirman estar mejor formados. Por el contrario, los estudios cualitativos que recogen la opinión de los empleadores (Barraycoa y Lasaga, 2009), evidencian que éstos consideran deficitaria la capacidad de trabajar en equipo los jóvenes titulados.

En segundo lugar, tanto empleadores como recién licenciados difieren en la concepción de esta competencia. Los estudiantes identifican el trabajo en equipo con el trabajo en grupo en tanto que para los empleadores significa algo más que una simple suma de esfuerzos.

Por todo ello se hace necesario abordar una definición que contemple todas las dimensiones de esta competencia, cuyo alcance es más complejo de lo que parece y, a su vez, ha de agrupar las perspectivas académicas y empresariales.

\section{REDEFINICIÓN DE LA COMPETENCIA TRABAJO EN EQUIPO}

En sentido genérico, podemos definir el trabajo en equipo como la capacidad de integrarse en grupos de trabajo para alcanzar objetivos comunes. En una primera 
aproximación pueden señalarse diferentes aspectos psicosociales que potencian esta competencia:

*Capacidad de integración. La integración ha de comportar una confluencia de sinergias regidas por un principio de efectividad, ya que los objetivos del equipo son más que la simple suma de objetivos individuales.

*Comunicación interpersonal. La consecución de los objetivos del equipo se verá condicionada no sólo por la capacidad de compartir conocimientos, sino también por la predisposición a escuchar y aceptar otros puntos de vista.

*Empatía. Los equipos de trabajo deben alcanzar un nivel óptimo de confianza y cohesión para su correcto funcionamiento. Para ello no es suficiente una integración mecánica de los individuos sino que las distintas habilidades personales deben ser compatibles.

*Capacidad para conseguir encultured knowledge. Capacidad de aprovechar los conocimientos y habilidades individuales para la potenciación del conocimiento grupal así como el reconocimiento de las metas grupales.

*Responsabilidad y compromiso. El individuo debe responsabilizarse de las tareas encomendadas por el equipo y comprometerse con el resultado del trabajo grupal.

*Toma de decisiones y gestión del tiempo. Para que el equipo funcione eficazmente sus componentes deben dominar los procesos de toma de decisiones y la temporalización de los objetivos.

En un sentido más específico, la consecución de esta competencia para un perfil de recién licenciado se optimiza con las siguientes habilidades:

*Reconocimiento de roles. En todo equipo de trabajo se conjugan diferentes roles. El recién licenciado ha de ser capaz de reconocerlos y de adaptarse al que se le asigne.

*Reconocimiento de liderazgo. Con frecuencia, el liderazgo es uno de los requisitos que garantiza el correcto funcionamiento de un equipo de trabajo. Difícilmente este rol recaerá en el recién licenciado, pero se espera de él la capacidad de reconocerlo y aceptarlo.

*Respeto al equipo. En la etapa de incorporación es importante que el egresado manifieste un especial respeto hacia los restantes integrantes del equipo. Aunque esta característica puede parecer evidente, los empleadores la tienen muy en cuenta. 


\section{DEL TRABAJO EN GRUPO AL TRABAJO EN EQUIPO}

En el decurso de una sesión de trabajo con empleadores acerca del papel de la educación en la adquisición de competencias (Barraycoa y Lasaga, 2009), un profesional afirmaba: "Nos encontramos con que los estudiantes creen que trabajan muy bien en equipo, ya que para ellos, según lo que se desprende de los procesos de selección, tienen claro que saben trabajar en equipo desde la Universidad. [Creen] que hacerlo en equipo es cuando cuatro personas hacen un trabajo, se lo reparten y luego 'cortan y pegan'." Esta autoimagen de los recién licenciados se debe a que a lo largo de la escolarización y de los estudios universitarios han realizado frecuentemente trabajos en grupo. Sin embargo, la estructura de trabajo de esta competencia en el mundo académico no se corresponde con los protocolos de actuación empresarial. Ello provoca una importante disonancia que las empresas se ven obligadas a enmendar en la primera etapa de su incorporación. Todo ello pone de relieve la necesidad de tender puentes, no sólo entre la Universidad y la Empresa, sino incorporando a las escuelas en este proceso de reflexión. Es imprescindible llegar a un consenso que favorezca una consonancia de las sinergias educativas para facilitar la incorporación al mundo laboral. En concreto, siendo la competencia de trabajo en equipo una de las más desarrolladas durante la etapa escolar, el consenso se hace imprescindible. La labor pedagógica debe encaminarse a que el alumno entienda que trabajar en equipo no es una mera adición de esfuerzos. Ha de fomentarse simultáneamente habilidades como la comunicación interpersonal, la responsabilidad y compromiso con los restantes miembros del equipo, la gestión del tiempo, la confianza y el respeto a la toma de decisiones grupales. Es importante que todos estos aspectos psicosociales se tomen en consideración al evaluar los trabajos en equipo.

\section{BIBLIOGRAFÍA}

- AA.VV. (2007): Informe Reflex: el profesional flexible en la sociedad del conocimiento en Observatorio Universitario de Inserción Laboral http:/ / www.insercionlaboral.net

- Barraycoa, J. y Lasaga, O., (2009). Competencias e inserción laboral: un análisis de la empleabilidad en los recién licenciados en Ade y Economía. Madrid. CEU Ediciones. http://www.insercionlaboral.net

- Fundación Carlos III (2005): Estudio sobre la identificación de los valores y competencias demandados en el mercado profesional a Titulados Universitarios. Servicio de Orientación y Planificación Profesional. Madrid. Universidad Carlos III. 
- Gómez Gras, J. M., et al.(Eds.) (2003): ¿Qué buscan las empresas en los universitarios? Cómo superar sus requisitos en Observatorio Universitario de Inserción Laboral http://www.insercionlaboral.net

- Pastor, M.S., et al. (2005): Las demandas sociales y su influencia en la planificación de las titulaciones en España en el marco del proceso de convergencia europea en educación superior. Madrid. Fundación Universidad Empresa. 